\title{
Erratum to: Stress and the nonsense-mediated RNA decay pathway
}

\author{
Alexandra E. Goetz ${ }^{1}$ - Miles Wilkinson ${ }^{1}$
}

Published online: 13 September 2017

(C) Springer International Publishing AG 2017

\section{Erratum to: Cell. Mol. Life Sci. DOI 10.1007/s00018-017-2537-6}

The original version of this article unfortunately contained errors in the section entitled "NMD in stress responses in plants".

The sentence, "As expected, Arabidopsis harboring mutations in these NMD genes are NMD-deficient, but they are viable and exhibit few defects under normal light and soil condition", is partially incorrect.

It should read as "Arabidopsis mutants with hypomorphic and null mutations in NMD genes do indeed exhibit NMD deficiency, but they typically have phenotypic defects, ranging from embryonic lethality to defects in shoot morphology, leaf morphology, cell cycle, meiotic progression, flowering, seed size, and seed germination. Only a few hypomorphic NMD mutant plants grow normally under normal lighting conditions".
The first paragraph of the section, second column, the pathovar Pseudomonas syringae was incorrectly claimed to be a virus; it is in fact a bacterial strain.

At the end of the first paragraph, second column, the sentence "While it is counter intuitive why the NMD pathway would reduce viral resistance..." should read "While it is counter intuitive why the NMD pathway would reduce bacterial immunity..." Various lines of evidence suggest that the elevated stress sensitivity of NMD-deficient plants is the result of their elevated and/or constitutive immune responses. This finding, coupled with other evidence, suggests that NMD controls the threshold for activation of innate immune response as a means to confer inducible bacterial defense.

The online version of the original article can be found under doi:10.1007/s00018-017-2537-6.

Miles Wilkinson

mfwilkinson@ucsd.edu

1 Department of Reproductive Medicine, School of Medicine, University of California San Diego, 9500 Gilman Dr.,

La Jolla 92093, USA 